\title{
Guest Editorial: Organic Light-Emitting Materials and Devices
}

\author{
Franky So a and Chihaya Adachib \\ a University of Florida, P.O. Box 116400, Gainesville, Florida 32611-6400 \\ ${ }^{\mathrm{b}}$ Kyushu University, Center for Future Chemistry, 744 Oaza Motooka, Nishi-Ku, \\ Fukuoka 819-0395, Japan
}

Organic light-emitting diodes (OLEDs), invented by Ching Wang Tang in 1987, were a scientific curiosity initially until Richard Friend's discovery of electroluminescence from polymers. Since then, a lot of active research activities have been undertaken to develop highly luminescent and charge-carrier-transporting organic materials, as well as efficient and stable OLEDs. In the last decade, progress has been made in this active area of research, and many companies like Samsung, Sony, and others began to commercialize OLEDs for flat-panel display applications. Compared to liquid crystal displays (LCDs), OLEDs offer many advantages, such as higher contrast ratio, higher response speed for video, and lower power consumption. Today, nextgeneration display technology based on OLEDs is a new enabling technology that promises to eventually replace LCD-based displays. Another promising application is in the area of solid-state lighting. In order to achieve this new goal, the performance of OLEDs needs to go to the next level, with higher efficiency and longer lifetime. Further development in highperformance materials such as efficient phosphorescent emitters, stable and high-bandgap host materials, and efficient carrier-transport materials are critical to the development of OLEDs with enhanced efficiency and long-term stability. In addition, further understanding of loss and device degradation mechanism(s) is also very important. In OLEDs, almost $75 \%$ of the photons generated are lost, and novel approaches to enhance light extraction are vital for solid-state lighting applications.

Papers that address the above issues and challenges are presented in this special section, which is based on presentations given at the 2010 conference on Organic Light-Emitting Materials and Devices at the SPIE Optics and Photonics meeting held in San Diego. We believe that readers will find the results of the studies discussed in these manuscripts informative, interesting, and in some cases unexpected or surprising. We hope that you will enjoy reading these papers.

1947-7988/2011/\$25.00 @ 2011 SPIE 